Int. J. Morphol.,

33(4): 1518-1524, 2015

\title{
Efectos Sobre el Comportamiento de Ratas Wistar Tratadas con el Compuesto Fenólico DM1
}

\author{
Behavioral Effects of the DM1 Phenolic Compound in Wistar Rats
}

Gonçalves, I. D."; Quincoces, J. A.*; Santos, R. P.**; dos Santos, G. A. A.*; Olave, E.*** \& Pardi, P.*

GONÇALVES, I. D.; QUINCOCES, J. A.; SANTOS, R. P.; DOS SANTOS, G. A. A.; OLAVE, E. \& PARDI, P. Efectos sobre cl comportamiento de ratas Wistar tratadas con el compuesto fenólico DM1. Int. J. Morphol., 33(4):1518-1524, 2015.

RESUMEN: El presente estudio tuvo como objetivo evaluar el efecto del compuesto fenólico polifuncional DM1 sobre el comportamiento motor, exploratório y ansiedad en ratas Wistar, analizadas en campo abierto (CA) y laberinto en cruz elevada (LCE). Se utilizaron 40 ratas Wistar adultas, divididas en 5 grupos (n= 8): Control (vehículo), DZP (Diazepam-2 mg/kg), DM1-150 mg/kg, DM1-300 mg/kg y DM1-450 mg/kg. Los animales fueron evaluados por un período de cinco minutos en CA y en el LCE, 30 min después de las administraciones (vía intraperitoneal). La evaluación en CA demostró reducción de la locomoción en los grupos DZP, DM1-300 y DM1-450 en relación al grupo control. Aumentó la locomoción en el grupo DM1-150 en relación al grupo DZPy disminuyó la locomoción en el grupo DM1-300 en relación al grupo DM1-150. Hubo disminución del levantar del grupo DZP en relación al grupo control. El grupo DM1-150 presentó aumento del levantar en relación al grupo DZP. Aumentó el tiempo estático (TE) en el grupo DZP y se redujo en el grupo DM1-300, ambos en relación al grupo control. El grupo DM1-150 presentó disminución del TE en relación al grupo DZP. La evaluación LCE presentó reducción del número de entradas en los brazos abiertos en el grupo DZP en relación al grupo control. Hubo reducción del número de entradas en los brazos cerrados en el grupo DZP en relación al grupo control y aumento de este parámetro en el grupo DM1-150 mg en relación al grupo DZP. Se redujó el número de cruzamientos entre los brazos cerrados en el grupo DZP en relación al grupo control. Los resultados en conjunto, sugieren que las dosis del compuesto fenólico polifuncional DM1 por sobre 150mg, tienen influencia en el estado emocional de los animales, indicando posible acción sedativa con probable inducción de relajamiento muscular.

PALABRAS CLAVE: Polifenoles; Campo abierto; Laberinto en cruz elevada; Comportamiento.

\section{INTRODUCCIÓN}

La utilización popular de plantas para el tratamiento de diversas enfermedades ha incentivado la búsqueda de nuevas sustancias con propiedades medicinales extraídas de vegetales. En los últimos años se han identificado varios compuestos que traen beneficio a la salud humana. Estas sustancias presentan un amplio espectro de actividad biológica debido a compuestos de naturaleza polifenólica en su composición, asociada principalmente con sus características antioxidantes.

Popularmente los vegetales con estas características se utilizan com antibacterianos, antiinflamatórios, antivirales y anticancerígenos.

La actividad biológica de los compuestos fenólicos en parte pueden ser explicados debido a su capacidad de modular la expresión genética, interfiriendo en la cascada de AMPc (3,5-adenosin-monofosfato-cíclico), segundos mensajeros en la transducción de señales e interacción con proteínas específicas que reducen la actividad de grupos e-aminos y la disponibilidad de determinados aminoácidos como la metionina, inhibiendo de esta forma la disponibilidad de determinados aminoácidos como la lisina y la destoxificación de cianuros (Soobrattee et al., 2005).

Varios estudios han correlacionado las actividades de los compuestos polifenólicos naturales con la reducción de determinados tumores malignos y de enfermedades cardiovasculares, por lo que se cree que estas propiedades estarían en directa relación con las propiedades antioxidantes de los compuestos fenólicos (Broinizi et al., 2007).

\footnotetext{
* Programa de Magister Profesional en Farmacia y Programa de Magister y Doctorado en Biotecnologia e Innovación en Salud, Universidade Anhanguera de São Paulo, São Paulo, Brasil.

** Universidade Federal do ABC, São Paulo, Brasil.

**** Facultad de Medicina, Universidad de La Frontera, Temuco, Chile.
} 
La actividad antioxidante de la curcumina puede inducir a la muerte celular por activación de la cascada apoptótica, debido a la producción de radicales superóxido (Piantino et al., 2009). La apoptosis en las células malignas ocurre por mecanismos dependientes o no, de las mitocondrias, donde la curcumina promueve la activación de la caspase 8 , disminución del potencial transmembrana mitocondrial, liberación de citocromo $\mathrm{C}$, secuencialmente la activación de la caspase-9 y caspase3 , que posteriormente inducirá a la fragmentación del ADN y muerte celular (Junior, 2009).

A pesar del amplio espectro de actividades biológicas, la utilización terapéutica de la curcumina es limitada. De este modo, surge la necesidad de una nueva metodologia que posibilite la producción de un compuesto fenólico semejante a la curcumina, aumentando su biodisponibilidad sistémica y por ende, las posibilidades de su aplicación terapéutica.

El objetivo del presente estudio fue desarrollar nuevas sustancias con propiedades terapéuticas equivalentes a los encontrados en los vegetales, intentando obtener un efectivo y potente compuesto fenólico que pueda utilizarse en procesos patológicos agudos y crónicos, y que además tenga una buena biodisponibilidad sistémica, baja toxicidad y buena tolerancia terapéutica.

Mediante los estudios que se han realizado, se desarrolló un compuesto con base en el principal compuesto fenólico de la Curcuma longa, la curcumina (([1,7bis(4-hidroxi-3-metoxi-fenil)-1,6-heptadiene-3,5diona]).Este compuesto análogo fue denominado como DM1 (4-[5-(4hidroxi-3-metoxi-fenil)-3-oxo-penta-1,4dienil]-2metoxi-fenolato de sódio). En estudios más específicos el compuesto fenólico DM1 presentó sorprendente actividad antitumoral, antiinflamatoria, analgésica y antiparasitaria (Quincocez et al., 2007).

Comportamiento de los compuestos fenólicos: Sabiendo que los compuestos fenólicos son sustancias que presentan amplio espectro de actividad biológica, este trabajo tiene como objetivo evaluar el efecto del compuesto fenólico polifuncional DM1 sobre el comportamiento de ratas evaluadas en los modelos de campo abierto (CA) y laberinto en cruz elevada (LCE).

Siendo la locomoción altamente estructurada y consistente, se presenta como un parámetro que puede ser cuantificado a través de la evaluación de la actividad general del comportamiento de animales de experimentación. Esta evaluación del comportamiento se presenta extremadamente importante para estudios en la áreas de la psicofarmacología y la psicobiologia (Kafkafi et al., 2003).

Los modelos de LCE y del test del CA son clásicos para la medición de la ansiedad a través de la evaluación del comportamiento general de animales (Belzung \& Griebel, 1999; Nadlewska et al., 2003; Prut \& Belzung, 2003; Lipkind et al., 2004; Hinojosa et al., 2006; Pritchard et al., 2008).

Estos modelos se basan en la evaluación del comportamiento exploratorio de animales expuestos a ambientes desconocidos o poco conocidos lo que promueve alteración en su comportamiento (Belzung \& Griebel). En este modelo es perfectamente posible evaluar parámetros altamente satisfactorios para estudios de fenómenos complejos como la ansiedad, debido a la posibilidad de evaluación del comportamiento espontáneo de los animales (Lipkind et al.; Eissa Ahmed et al., 2009).

Debido a que la locomoción presenta una correlación directa con la ansiedad, es perfectamente posible provocar alteraciones del comportamiento a través de la utilización de sustancias, por ejemplo, con actividades ansiolíticas, lo que llevaría a los animales a aumentar el período de exploración en el perímetro central del CA, comportamiento inusitado, debido a que esta región representa una amenaza para el animal (Lipkind et al.; Sousa et al., 2006).

Modelos animales válidos para ansiedad y depresión desempeñan diferentes estímulos de aversión en tareas del comportamiento, lo cual puede ser relacionado con la exploración y aversión a áreas abiertas y claras en el CA, aprovechando la tendencia natural de la rata para la exploración (Barbosa, 2006).

La medida del nivel de ansiedad puede ser correlacionada al tiempo usado en que el animal permanece con los brazos abiertos (BA) y cerrados (BC), cuando son evaluados en el modelo del LCE. De la misma forma, la correlación entre el tiempo de permanencia de los animales a lo largo de las paredes y en el perímetro central, en el modelo de CA pueden ser consideradas medidas de ansiedad. El LCE es considerado un instrumento seguro para evaluación tanto de la emocionalidad como de la ansiedad (Avital et al., 2001; Corrobrez \& Beroglio, 2005; Pritchard et al.).

El objetivo de este trabajo fue evaluar el comportamiento de ratas Wistar tratadas con diferentes dosis de compuesto fenólico polifuncional DM1 (4-[5-(4hidrixi-3-metoxifenil)-3-oxo-penta-1,4-dienil]-2-metoxi-fenolato de sódio) en los modelos de CA y en el LCE. 


\section{MATERIAL Y MÉTODO}

Se utilizaron 40 ratas Wistar, machos, adultos con 90 días de edad. Los animales fueron utilizados en conformidad con las normas y procedimientos éticos vigentes para experimentación animal, estudio que fue previamente sometido y aprobado por el Comité de Etica de La Universidad de Anhanguera de São Paulo - UNIAN, São Paulo Protocolo No 20090171. Los animales fueron mantenidos en cajas de polipropileno de $41 \times 34 \times 16 \mathrm{~cm}$, y en cada una de ellas se colocaron 4 ratas, mantenidas en el Laboratorio de Farmacología Experimental, con temperatura y humedad controladas y fotoperíodo de $12 \mathrm{~h}$. Las ratas fueron alimentadas con ración padrón y agua ad libitum. Se identificaron, pesaron y divididos aleatoriamente en 5 grupos $(\mathrm{n}=8)$ : Control (CONT. vehículo - água), DZP (Diazepan $2 \mathrm{mg} / \mathrm{Kg}$ ), DM1-150 (150 mg/kg), DM1-300 (300 mg/kg) y DM1-450 $(450 \mathrm{mg} / \mathrm{kg}$ ). Todos los grupos recibieron sus respectivas sustancias y dosis por vía intraperitoneal.

Las evaluaciones fueron realizadas 30 min después de la administraciones y se consideraron los siguientes parámetros en el CA: locomoción (LO), tiempo en que se mantuvo estático (TE), tiempo de permanencia en el perímetro central (TC), tiempo de permanencia en el perímetro lateral (TL) y tiempo de aseo (Grooming) (TG).

En el LCE se evaluó: tiempo de permanencia en los brazos abiertos, (TBA), tiempo de permanencia en los brazos cerrados (TBF), número de entradas en los brazos abiertos (NBA), número de entradas en los brazos cerrados (NBC) y número de cruzamientos entre los brazos cerrados (NC).

Los datos obtenidos fueron agrupados, tabulados y analizados estadísticamente a través del software GraphPad Instat v 3.01. En todos los tests aplicados, el nível de significación fue de 5\%, o sea, la probabilidad p <0,05 fue considerada capaz de revelar diferencias estadísticamente significativas entre los grupos de animales estudiados.

\section{RESULTADOS}

Evaluación del comportamiento en el modelo de CA. En la Figura 1 se observa la comparación entre los grupos para evaluación del comportamiento en relación al número de movimientos en el modelo de CA, donde se presentó una reducción $(\mathrm{p}<0,001)$ de la locomoción en los grupos DZP y DM1-300; disminución $(\mathrm{p}<0,05)$ de la LO en el grupo DM1-450, todos en relación al grupo control; disminución $(\mathrm{p}<0,001)$ de la locomoción en el grupo DM1300 en relación al grupo DM1-150. También hubo aumento $(\mathrm{p}<0,001)$ de la locomoción del grupo DM1-150mg en relación al grupo DZP. No hubo diferencias estadísticamente significativas del grupo DM1-150 en relación al grupo control.

La Tabla I presenta la reducción $(\mathrm{P}<0,001)$ de la locomoción en los grupos DZP (Diazepam $2 \mathrm{mg} / \mathrm{kg}$ ) y DM1-300 mg. Disminución ( $<<0,05)$ de la locomoción del grupo DM1-450 mg, todos en relación al grupo control. Se observó disminución $(\mathrm{P}<0,001)$ de la locomoción del grupo DM1-300 mg en relación al grupo DM1-150. Este último grupo presentó aumento $(\mathrm{P}<0,001)$ de la locomoción en relación al grupo Diazepam (DZP 2mg/kg).

Los animales del grupo Diazepam presentaron disminución $(\mathrm{P}<0,05)$ del acto de levantar sobre los miembros posteriores en relación al grupo control. El grupo DM1-150 mg presentó aumento $(\mathrm{P}<0,001)$ del parámetro levantar en relación al grupo Diazepam.

El grupo Diazepam presentó aumento $(\mathrm{P}<0,05)$ del TE en relación al grupo control. El grupo DM1-300 mg presentó reducción $(\mathrm{P}<0,05)$ del $\mathrm{TE}$ en relación al grupo control. También se observó disminución $(\mathrm{P}<0,05)$ del TE en el grupo DM1-150 mg en relación al grupo Diazepam. No se observaron alteraciones entre los otros parámetros evaluados.

Tabla I. Evaluación comportamental en el campo abierto (Promedios y DE).

\begin{tabular}{lcccccc}
\hline & LO & LE & TP & TL & TC & TG \\
\hline CONT & $117 \pm 28$ & $36 \pm 15$ & $4 \pm 6$ & $265 \pm 19$ & $33 \pm 22$ & $22 \pm 22$ \\
DZP - 2 mg & $44 \pm 38 \mathrm{~A}$ & $7 \pm 8^{\mathrm{D}}$ & $129 \pm 73^{\mathrm{E}}$ & $206 \pm 112$ & $94 \pm 12$ & $10 \pm 11$ \\
DM1 - 150 mg & $112 \pm 40 \mathrm{~B}$ & $44 \pm 27 \mathrm{~B}$ & $9 \pm 15 \mathrm{~F}$ & $246 \pm 48$ & $54 \pm 48$ & $30 \pm 29$ \\
DM1 - 300 mg & $52 \pm 29^{\mathrm{AC}}$ & $16 \pm 13$ & $96 \pm 88 \mathrm{D}$ & $230 \pm 98$ & $68 \pm 99$ & $18 \pm 16$ \\
DM1 - 450 mg & $64 \pm 35 \mathrm{D}$ & $22 \pm 12$ & $58 \pm 64$ & $266 \pm 19$ & $34 \pm 19$ & $9 \pm 9$ \\
\hline
\end{tabular}

$\mathrm{A}=\mathrm{P}<0,001$ reducción en relación al grupo Control (Análisis de Varianza - ANOVA)

$\mathrm{B}=\mathrm{P}<0,001$ aumento en relación al grupo Diazepam-2 mg (Análisis de varianza-ANOVA)

$\mathrm{C}=\mathrm{P}<0,001$ reducción en relación al grupo DM1-150 mg (Análisis de varianza - ANOVA)

$\mathrm{D}=\mathrm{P}<0,05$ reducción en relación al grupo control (Análisis de varianza - ANOVA)

$\mathrm{E}=\mathrm{P}<0,05$ aumento en relación al control (Análisis de varianza - ANOVA)

$\mathrm{F}=\mathrm{P}<0,05$ reducción en relación al grupo Diazepam-2 mg (Análisis de varianza - ANOVA) 


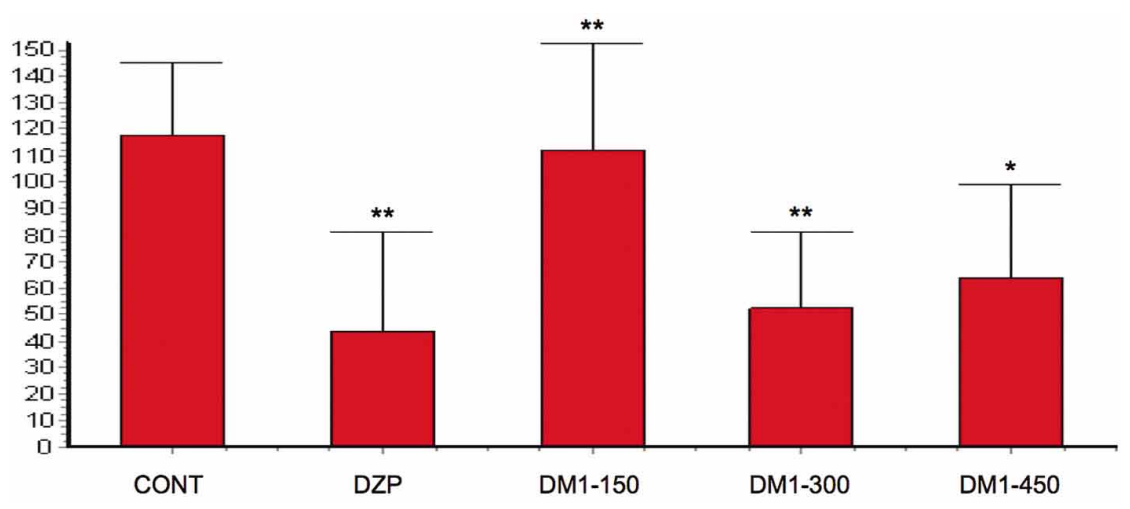

Fig. 1. Comparación entre grupos para evaluación del comportamiento en relación al número de movimientos en el CA.

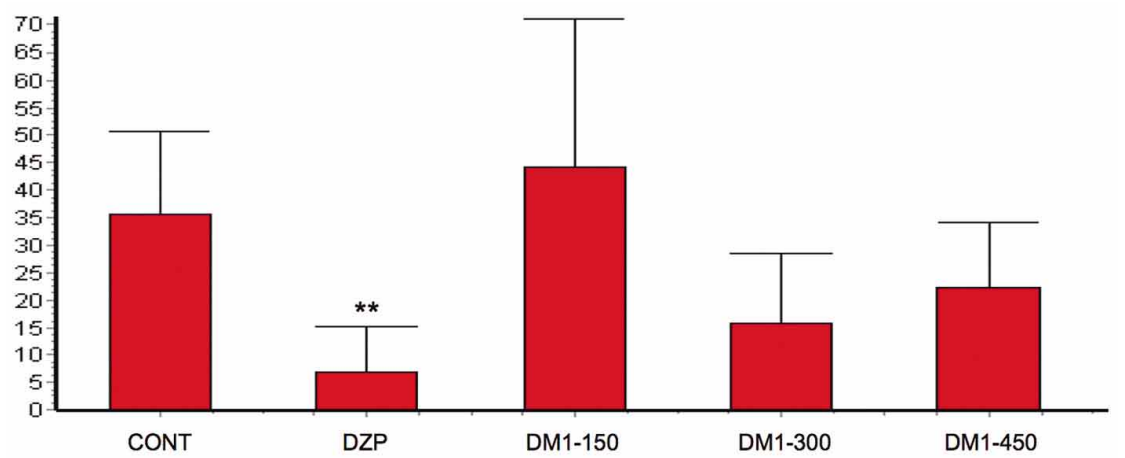

Fig. 2. Comparación entre grupos para evaluación del comportamiento en relación al número de exploración aérea (levantar) en el CA.

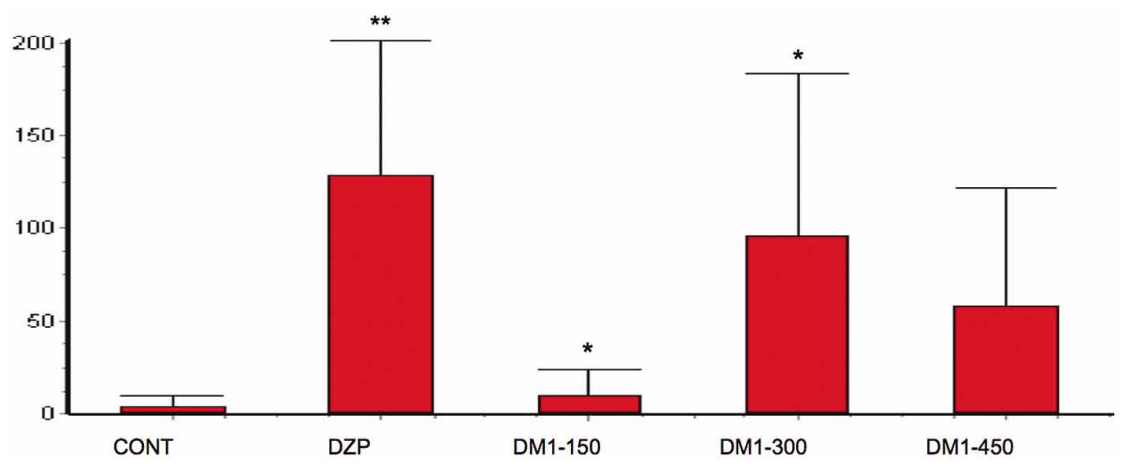

Fig. 3. Comparación entre grupos para evaluación del comportamiento en relación al TE en el CA.

En la Figura 2 se muestra una comparación entre los grupos para evaluación del comportamiento en relación al número de exploración aérea (LE) en el modelo de campo abierto. Se presentó reducción $(\mathrm{p}<0,05)$ del parámetro levan$\operatorname{tar}(\mathrm{LE})$ en el grupo DZP en relación al grupo control y aumento $(\mathrm{P}<0,001)$ del levantar en el grupo DM1-150 mg en relación al grupo Diazepam (DPZ-2 mg/ $\mathrm{kg})$.

En la Figura 3 se muestra la comparación entre los grupos para evaluación del comportamiento en relación al TE en el modelo de CA. Se presentó aumento $(\mathrm{p}<0,001)$ del TE en el grupo DZP y aumento $(\mathrm{P}<0,05)$ en el grupo DM1-300 mg, ambos en relación al grupo control. También presentó disminución $(\mathrm{p}<0,001)$ del TE en el grupo DM1-150mg en relación al grupo DZP-2 mg/kg.

Evaluación del comportamiento en el modelo de LCE. En la Figura 4 se presenta reducción estadísticamente significativa $(\mathrm{p}<0,001)$ del número de entradas en los brazos abiertos (NBA) del grupo Diazepam (DZP) en relación al grupo control.

En la Figura 5 se presenta la evaluación del NBC en el modelo LCE. Se presentó reducción $(\mathrm{p}<0,001)$ del NBC del grupo Diazepam (DZP) en relación al grupo control. Se pudo observar también un aumento $(p>0,005)$ del NBC del grupo DM1-150 mg en relación al grupo Diazepam (DZP-2 m/kg). No se observaron alteraciones estadísticamente significativas entre los demás grupos evaluados.

En la Figura 6 se presenta una reducción estadísticamente significativa $(\mathrm{p}<0,001)$ del número de cruzamientos (NC) en el grupo DZP en relación al grupo control. No se observaron alteraciones estadísticamente significativas en la evaluación entre los demás grupos.

La Tabla II muestra la comparación general entre los grupos CONTROL, DZP; DM1-150, DM1300; DM1-450 y DM1-600, donde la evaluación comportamental en el modelo de LCE presentó reducción estadísticamente significativa $(\mathrm{p}<0,001)$ del NBA del grupo DZP en relación al grupo control. En esta evaluación se observó reducción $(p<0,001)$ del NBC del grupo DZP en relación al grupo control y un aumento $(p<0,05)$ de este parámetro en el grupo DM1-150 mg en relación al grupo DZP. Esta evaluación demostró reducción $(\mathrm{p}<0,001)$ del NC en el grupo DZP en relación al grupo control. 


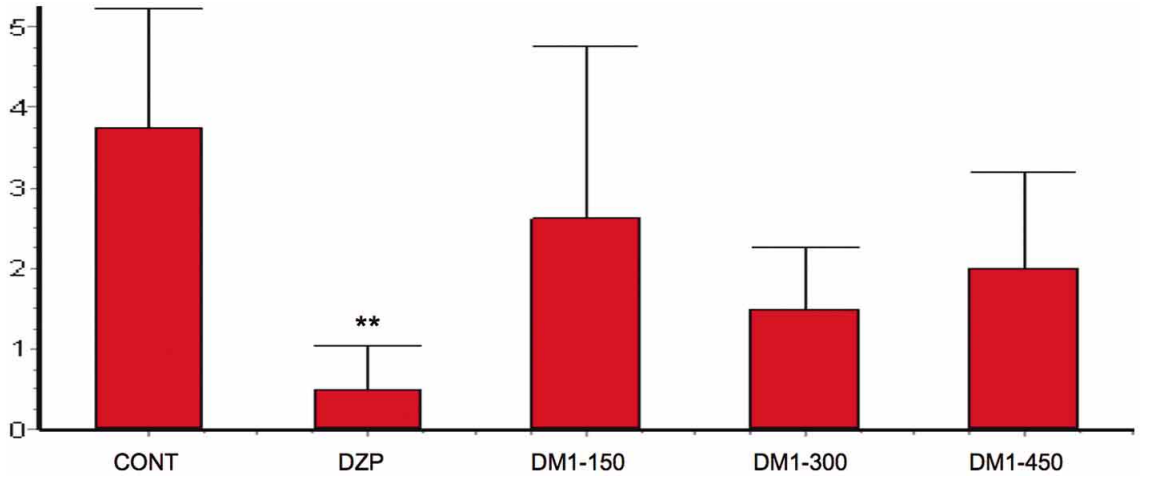

Fig. 4. Comparación entre grupos para evaluación del número de entradas en los brazos abiertos en el LCE.

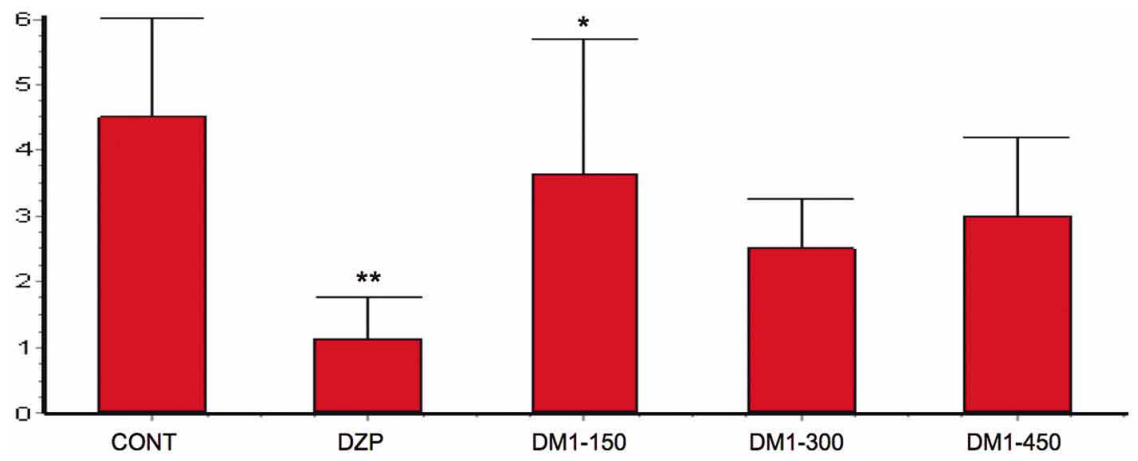

Fig. 5. Comparación entre grupos para evaluación del número de entradas en los brazos cerrados en el LCE.

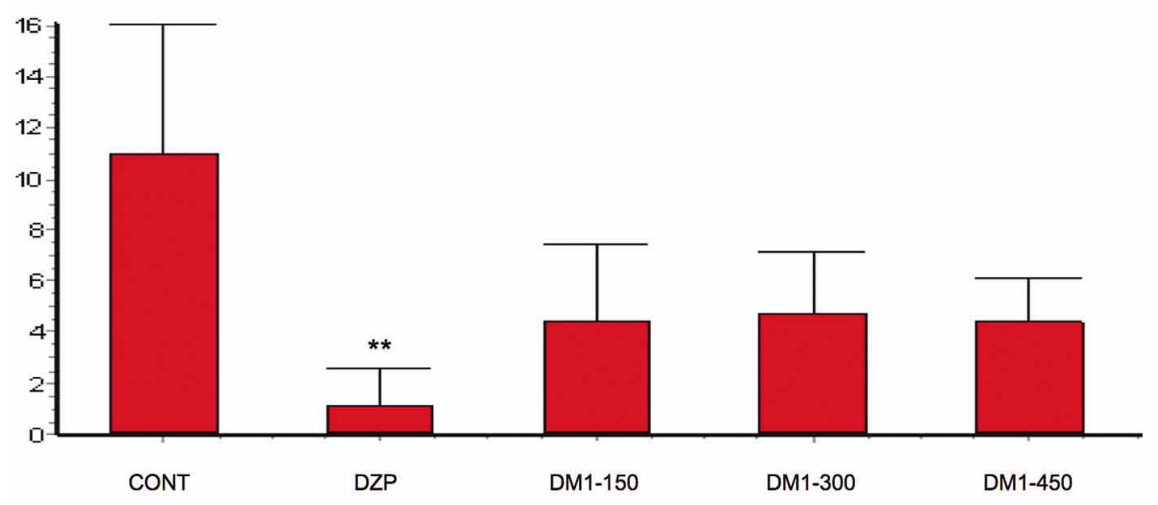

Fig. 6. Comparación entre grupos para evaluación del número de cruzamientos en el LCE.

\section{DISCUSIÓN}

Las redes neuronales innatas son responsables por la coordinación del comportamiento y de la interacción con el ambiente. Esta región cerebral está íntimamente ligada al control del aumento de la actividad exploratória de ratas evaluadas en campo abierto (Zangrossi \& File, 1992). Así, a través de la actividad motora del animal expuesto a un ambiente nuevo es posible evaluar parámetros relacionados a la respuesta de ansiedad y de miedo (Piazza et al., 1993; Kabbaj et al., 2000).

Los comportamientos de locomoción y levantar son relacionados positivamente con las alteraciones observadas en la actividad motora y exploratoria que los animales presentan a consecuencia de un estado emocional alterado (Godinho et al., 2002).

El análisis de los resultados obtenidos en campo abierto demostró disminución de la LO en los grupos Diazepam, DM1-300 y DM1450 en relación al grupo control. Se observó reducción de la LO en el grupo DM1-300 en relación al grupo DM1-150. También se observó aumento de la LO de los animales en el grupo DM1-150 con respecto al grupo Diazepam y aumento del TE de los grupos Diazepam y DM1300, ambos en relación al grupo control. Hubo disminución del TE de los animales del grupo DM1-150 en relación al grupo Diazepam.

Tabla II. Evaluación del comportamiento en el laberinto en cruz elevada (PromediosDE).

\begin{tabular}{lccccc}
\hline & TBA & TBF & NBA & NBF & NC \\
\hline CONT & $106 \pm 54$ & $314 \pm 54$ & $3,7 \pm 1,5$ & $4,5 \pm 1,5$ & $11 \pm 5$ \\
DZP - 2 mg & $169 \pm 185$ & $243 \pm 180$ & $0,5 \pm 0,5 \mathrm{~A}$ & $1,1 \pm 0,6^{\mathrm{A}}$ & $1 \pm 1^{\mathrm{A}}$ \\
DM1 - 150 mg & $108 \pm 69$ & $312 \pm 69$ & $2,6 \pm 2,1$ & $3,6 \pm 2,1^{\mathrm{B}}$ & $4 \pm 3$ \\
DM1 - 300 mg & $63 \pm 21$ & $357 \pm 21$ & $1,5 \pm 0,8$ & $2,5 \pm 0,8$ & $5 \pm 2$ \\
DM1 - 450 mg & $113 \pm 79$ & $306 \pm 79$ & $2,0 \pm 1,2$ & $3,0 \pm 1,2$ & $4 \pm 2$ \\
\hline
\end{tabular}


Probablemente, la disminución de la LO del grupo Diazepam en el modelo de CA esté relacionada a los efectos colaterales producidos por el Diazepam, entre los cuales, la sedación y el relajamiento muscular llevaría a una reducción de la LO y del levantar. Se aumenta el período de TE y se disminuye el tiempo de exploración aérea (Prut \& Belzung).

La reducción de la LO y el aumento del TE detectados en los animales que recibieron el compuesto DM1 en dosis de 300 y $450 \mathrm{mg} / \mathrm{kg}$ y evaluados con el test de CA, confirman la interacción de este compuesto sobre la emocionalidad de los animales en estudio.

El aumento de la LO en los animales del grupo DM1150 en relación al grupo Diazepam, no representa directamente una disminución de la ansiedad o aumento de la actividad, porque estos animales no presentaron diferencias estadísticamente significativas en relación al grupo control. La significación estadística puede ser explicada por la disminución de la LO de los animales tratados con Diazepam, los cuales presentaron disminución de la actividad exploratória.

La activación del sistema de inhibición cerebral promueve la reducción o la inhibición de las actividades del animal, pudiendo llegar a casos extremos der inmovilidad o congelamiento (Papez, 1937). El aumento del TE es un parámetro indicativo de un alto nível de stress (Prut \& Belzung; Sachetti et al.) como respuesta a estímulos ambientales, entre los cuales está la incorporación de estos animales en un ambiente desconocido (Cloninger et al., 1993; Svradic et al., 2002).

El aumento del TE en el grupo DM1-300 en relación al grupo control, puede sugerir una actividad sedativa de estos animales, posiblemente por la acción de dosis más elevadas del compuesto fenólico DM1. Los animales que recibieron dosis de $150 \mathrm{mg} / \mathrm{kg}$ de DM1 no presentaron diferencias estadísticamente significativas en relación al grupo control.

La disminución de este parámetro puede presentar una relación directa con la disminución del stress de las ratas evaluadas en CA. De esta forma, se puede observar que el Diazepam provocó una disminución del stress de estos animales, por este motivo los del grupo Diazepam presentaron menor exploración aérea (levantar), explicando de este modo, el aumento del parámetro levantar en los animales del grupo DM1-150 en relación al grupo Diazepam.

Drogas con efectos estimulantes motores pueden presentar aumento del parámetro levantar (Pellow et al., 1985). Tal afirmación no ocurrió con los animales del grupo DM1-150, no presentando diferencias estadísticamente significativas respecto al grupo control, siendo ésta la dosis máxima ideal para uso terapéutico, ya que no produce alteraciones de comportamiento.
Comparando las diferentes dosis del compuesto fenólico DM1 en el LCE, se observó aumento del NBC en los animales del grupo DM1-150 en relación al grupo Diazepam. $\mathrm{Al}$ contrario, los animales del grupo Diazepam presentaron reducción del número de entradas, tanto en NBA como en NBC del laberinto en cruz elevada. El grupo Diazepam también presentó reducción del número de cruzamientos entre los brazos cerrados del laberinto, todos en relación al grupo control.

Posiblemente, la disminución de los parámetros mencionados, estén relacionados con los efectos sedativos y de relajamiento muscular producidos por el Diazepam (Prut \& Belzung).

La actividad sedativa puede ser definida por la reducción de la actividad locomotora y disminución del nível de vigilancia. El efecto hipnótico, diferente de la sedación, es capaz de inducir y mantener el sueño por cierto período de tiempo, características de los benzodiazepínicos que poseen propiedades ansiolíticas y sedativas, utilizados con frecuencia para reducir síntomas físicos de la ansiedad (Souza et al.).

En nuestro estudio, el compuesto fenólico DM1 en dosis de 300 y $450 \mathrm{mk} / \mathrm{kg}$ redujeron de forma significativa la LO en relación a los grupos control y DM1-150. Estos datos sugieren que el DM1 en dosis de 300 y $450 \mathrm{mg} / \mathrm{kg}$ interfieren en las emociones y en el actividad exploratória de los animales. En conjunto, los resultados del comportamiento demostraron que el compuesto fenólico DM1 presenta un importante efecto sedativo, aunque su mecanismo de acción aún no ha sido esclarecido. Es necesario continuar investigando para esclarecer algunas interrogantes que aún persisten.

GONÇALVES, I. D.; QUINCOCES, J.A.; SANTOS, R. P.; DOS SANTOS, G. A. A.; OLAVE, E. \& PARDI, P. Behavioral effects of the DM1 phenolic compound in wistar rats. Int. J. Morphol., 33(4):1518-1524, 2015.

SUMMARY: This study aimed to evaluate the effect of polyfunctional phenolic compound DM1 on the motor behavior, exploratory and anxiety in Wistar rats tested in open field (OF) and in elevated plus-maze (EPM). We used 40 adult Wistar rats divided in 5 groups $(\mathrm{n}=8)$ : Control (vehicle), DZP (diazepam-2 $\mathrm{mg} / \mathrm{kg}$ ), DM1-150 mg/kg, DM1-300 mg/kg and DM1-450 mg/kg. The animals were evaluated for a period of five minutes in the $\mathrm{OF}$ and EPM, 30 min after administrations (intraperitoneally). The evaluation in OF showed reduction in the locomotion in the DZP, DM1-300 and DM1-450 groups relative to the control group. It increased locomotion in DM1-150 group relative to the DZP group and decreased locomotion in DM1-300 group relative to the group DM1-150. There was decrease of the lifting action in the DZP group relative to the control group. The DM1-150 group presented increase of the lifting action compared to DZP group. It increased the static time (ST) in the DZP group and decreased in the DM1300 group, both in relation to the control group. The DM1-150 
group presented decrease of the ST compared to DZP group. The EPM evaluation presented reducing the number of entries into the open arms in the DZP group relative to the control group. There was reduction in the number of entries into the closed arms in the DZP group relative to the control and increase of this parameter in the DM1-150 group in relation to DZP group. The number of crossings between the closed arms in the DZP group relative to the control group decreased. The overall results suggest that the dose of polyfunctional phenolic compound DM1 above $150 \mathrm{mg}$ have influence on the emotional state of the animals, indicating possible sedative action likely induction of muscle relaxation.

KEY WORDS: Polyphenols; Open field; Elevated plus maze; Behavior.

\section{REFERENCIAS BIBLIOGRÁFICAS}

Avital, A.; Richter-Levin, G.; Leschiner, S.; Spanier, I.; Veenman, L.; Weizman, A. \& Gavish, M. Acute and repeated swim stress effects on peripheral benzodiazepine receptors in the rat hippocampus, adrenal, and kidney. Neuropsychopharmacology, 25(5):669-78, 2001.

Barbosa, C. P. C. Efeito da atividade fisica no comportamento de ratos Wistar expostos ao diazepam durante a lactação. Dissertação (Mestrado). São Paulo, Universidade Bandeirante de São Paulo - UNIBAN, 2006.

Belzung, C. \& Griebel, G. Measuring normal and pathological anxiety-like behaviour in mice: a review. Behav. Brain Res., 125(1-2):141-9, 2001.

Broinizi, P. R. B.; Andrade-Wartha, E. R. S.; Silva, A. M. O.; Novoa, A. J. V.; Torres, R. P.; Azeredo, H. M. C.; Alves, R. E. \& Mancini-Filho, J. Avaliação da atividade antioxidante dos compostos fenólicos naturalmente presentes em subprodutos do pseudofruto de caju (Anacardium occidentale L.). Ciênc. Tecnol. Aliment., 27(4):902-8, 2007.

Cloninger, C. R.; Svrakic, D. M. \& Przybeck, T. R. A psychobiological model of temperament and character. Arch. Gen. Psychiatry, 50(12):975-90, 1993.

Corobrez, A. P. \& Bertoglio, L. J. Ethological and temporal analyses of anxietylike behavior: the elevated plus-maze model 20 years on. Neurosci. Biobehav. Rev., 29(8):1193-205, 2005.

Eissa Ahmed, A. A.; Al-Rasheed, N. M. \& Al-Rasheed, N. M. Antidepressantlike effects of rosiglitazone, a PPARg agonist, in the rat forced swim and mouse tail suspension tests. Behav. Pharmacol., 20(7):635-42, 2009.

Godinho, A. F.; Trombini, T. V. \& Oliveira, E. C. Effects of elevated calcium on motor and exploratory activities of rats. Braz. J. Med. Biol. Res., 35(4):451-7, 2002.

Hinojosa, F. R.; Spricigo, L. Jr.; Izidio, G. S.; Brüske, G. R.; Lopes, D. M. \& Ramos, A. Evaluation of two genetic animal models in behavioral tests of anxiety and depression. Behav. Brain Res., 168(1):127-36, 2006.

Junior, J. F. Curcumina e Câncer: antiproliferativo, antiapoptótico, antiangiogénico e antimetastático. Campo Grande, Portal Educação, 2009. Disponible en: http://www.portaleducacao.com.br/

Kabbaj, M.; Devine, D. P.; Savage, V. R. \& Akil, H. Neurobiological correlates of individual differences in novelty-seeking behavior in the rat: differential expression of stress-related molecules. J. Neurosci., 20(18):6983-8, 2000.
Kafkafi, N.; Lipkind, D.; Benjamini, Y.; Mayo, C. L.; Elmer, G. I. \& Golani, I. SEE locomotor behavior test discriminates C57BL/6J and DBA/2J mouse inbred strains across laboratories and protocol conditions. Behav. Neurosci., 117(3):464-77, 2003.

Lipkind, D.; Sakov, A.; Kafkafi, N.; Elmer, G. I.; Benjamini, Y. \& Golani, I. New replicable anxiety-related measures of wall vs center behavior of mice in the open field. J. Appl. Physiol. (1985), 97(1):347-59, 2004.

Nadlewska, A.; Car, H.; Wisniewska, R.; Hoy, Z. \& Wisniewski, K. Behavioral effects of D-AP7 in rats subjected to experimental hypoxia. Pol. J. Pharmacol., 55(3):337-44, 2003.

Papez, J. W. A proposed mechanism of emotion. Arch. Neurol. Psychiatry, 38(4):725-43, 1937.

Pellow, S.; Chopin, P.; File, S. E. \& Briley, M. Validation of open:closed arm entries in an elevated plus-maze as a measure of anxiety in the rat. $J$. Neurosci. Methods, 14(3):149-67, 1985.

Piazza, P. V.; Deroche, V.; Deminière, J. M.; Maccari, S.; Le Moal, M. \& Simon, $\mathrm{H}$. Corticosterone in the range of stress-induced levels possesses reinforcing properties: implications forsensation-seeking behaviors. Proc. Natl. Acad. Sci. USA, 90(24):11738-42, 1993.

Piantino, C. B.; Salvadori, F. A.; Ayres, P. P.; Kato, R. B.; Srougi, V.; Leite, K. R. \& Srougi, M. An evaluation of the anti-neoplastic activity of curcumin in prostate cancer cell lines. Int. Braz. J. Urol., 35(3):354-60, 2009.

Pritchard, L. M.; Van Kempen, T. A.; Williams, H. \& Zimmerberg, B. Introductory Neuroscience Curse Demonstrating Effects of Housing Environment on Anxiety an Psycho stimulant Sensitivity. J. Undergrad. Neurosci. Educ., $7(1): 26-32,2008$.

Prut, L. \& Belzung, C. The open field as a paradigm to measure the effects of drugs on anxiety-like behaviors: a review. Eur. J. Pharmacol., 463(1-3):333, 2003.

Quincoces, J.; Maria, D. A.; Rando, D. G.; Pardi, P. C.; Martins, C. A. S. \& Souza, P. O. Patente brasileira número: PI No 0602640-0, (06.07.2006) PCT/BR 2007/000175.

Soobrattee, M. A.; Neergheen, V. S.; Luximon-Ramma, A.; Aruoma, O. I. \& Bahorun, T. Phenolics as potential antioxidant therapeutic agents: mechanism and actions. Mutat. Res., 579(1-2):200-13, 2005.

Souza, J. P.; Nogueira, G. M.; Mataqueiro, M. I. \& Queiroz-Neto, A. Atividade colinesterásica cerebral e comportamento de ratos após exposição perinatal ao diclorvós. Ciênc. Rural, 36(2):501-8, 2006.

Svradic, D. M.; Draganic, S.; Hill. K.; Bayon, C.; Przybeck, T. R. \& Cloninger, C. R. Temperament, character, and personality disorders: etiologic, diagnostic, treatment issues. Acta Psychiatr. Scand., 106(3):189-95, 2002.

Zangrossi, H. Jr. \& File, S. E. Behavioral consequences in animal tests of anxiety and exploration of exposure to cat odor. Brain Res Bull., 29(3-4):381-8, 1992.

\section{Dirección para correspondencia:}

Ivair D. Gonçalves - Prof. Dr. Paulo C. Pardi

Programa de Mestrado e

Doutorado em Biotecnologia e Inovação em Saúde

Universidade Anhanguera de São Paulo

Rua Maria Cândida, 1813.

São Paulo, SP 02071-013

BRASIL

Received: 15-07-2015

Accepted: 22-10-2015

Email: ivairdg@yahoo.com.br, paulopardi@hotmail.com 